\title{
Effect of magnetic boundary conditions on the dynamo threshold of von Kármán swirling flows
}

\author{
C. Gissinger ${ }^{1,2}$, A. Iskakov ${ }^{3,4}$, S. Fauve ${ }^{1}$, E. Dormy ${ }^{2,3}$ \\ 1' Laboratoire de Physique Statistique, École Normale Supérieure CNRS, 24 rue Lhomond, F-75005 Paris (France). \\ Laboratoire de Radioastronomie, École Normale Supérieure CNRS, 24 rue Lhomond, F-75005 Paris (France). \\ Institut de Physique du Globe de Paris (France). \\ U.C.L.A., Physics Department (U.S.A.).
}

PACS 91.25. Cw - Origins and models of the magnetic field; dynamo theories

PACS $47.65 .+\mathrm{a}$ - Magnetohydrodynamics and electrohydrodynamics

\begin{abstract}
We study the effect of different boundary conditions on the kinematic dynamo threshold of von Kármán type swirling flows in a cylindrical geometry. Using an analytical test flow, we model different boundary conditions: insulating walls all over the flow, effect of sodium at rest on the cylinder side boundary, effect of sodium behind the impellers, effect of impellers or side wall made of a high magnetic permeability material. We find that using high magnetic permeability boundary conditions decreases the dynamo threshold, the minimum being achieved when they are implemented all over the flow.
\end{abstract}

Dynamo action, i.e., self-generation of magnetic field by the flow of an electrically conducting fluid, is at the origin of planetary, stellar and galactic fields [1]. Fluid dynamos have been observed only recently in laboratory experiments in Karlsruhe [2] and Riga [3] by geometrically constraining the flow lines in order to mimic laminar flows that were known analytically for their dynamo effi- ciency [4]. More recently, the VKS experiment displayed self-generation in a less constrained geometry, e.g. a vion Kármán swirling flow generated between two counterrotating impellers in a cylinder [5]. However, until now, dynamo action in the VKS geometry has been found only when the impellers are made of soft iron. It is thus of primary importance to understand how the dynamo problem is modified by the presence of magnetic material at the flow boundaries. We address this problem here using a kinematic dynamo code in a cylindrical geometry. Two important approximations are made to simplify the study. First, an analytic test flow that mimics the geometry of the mean flow of the VKS experiment is considered. Second, the magnetic boundary conditions are taken in the limit of infinite magnetic permeability of the boundaries compared to the one of the fluid. This seems a reasonable approximation for soft iron compared to liquid sodium. Our main result is that the critical magnetic Reynolds number, $R m_{c}$, for dynamo generation is significantly decreased with boundaries of high magnetic permeability all over the flow.

The VKS experimental set-up is sketched in Figure 1. A turbulent von Kármán flow of liquid sodium is generated by two counter-rotating impellers (rotation frequencies $F_{1}$ and $F_{2}$ ). The impellers are made of iron disks of radius $154 \mathrm{~mm}$, fitted with 8 iron blades of height $41.2 \mathrm{~mm}$, and are placed $371 \mathrm{~mm}$ apart in an inner cylinder of radius $206 \mathrm{~mm}$ and length $524 \mathrm{~mm}$. It is surrounded by sodium at rest in another concentric cylindrical vessel, $578 \mathrm{~mm}$ in inner diameter. This has been shown to decrease the dynamo threshold in kinematic computations based on the mean flow velocity [6]. When the impellers are operated at equal and opposite rotation rates $F$, a statistically stationary magnetic field is generated above a magnetic Reynolds number $R_{m} \sim 30$ [5]. The large scale field involves an azimuthal component and a poloidal one which is dominated by an axial dipole. This geometry has been understood with a simple $\alpha-\omega$ dynamo model [7] by taking into account the helical nature of the flow that is ejected by the centrifugal force close to each impeller between successive blades. Relying on the mean flow alone to compute the kinematic dynamo, smoothes out these non axisymmetric velocity fluctuations and thus cannot generate an axisymmetric field according to Cowling theorem. A non axisymmetric field is obtained, dominated by an equatorial dipole $[6,8]$.

When the disks are counter-rotating at the same fre- 


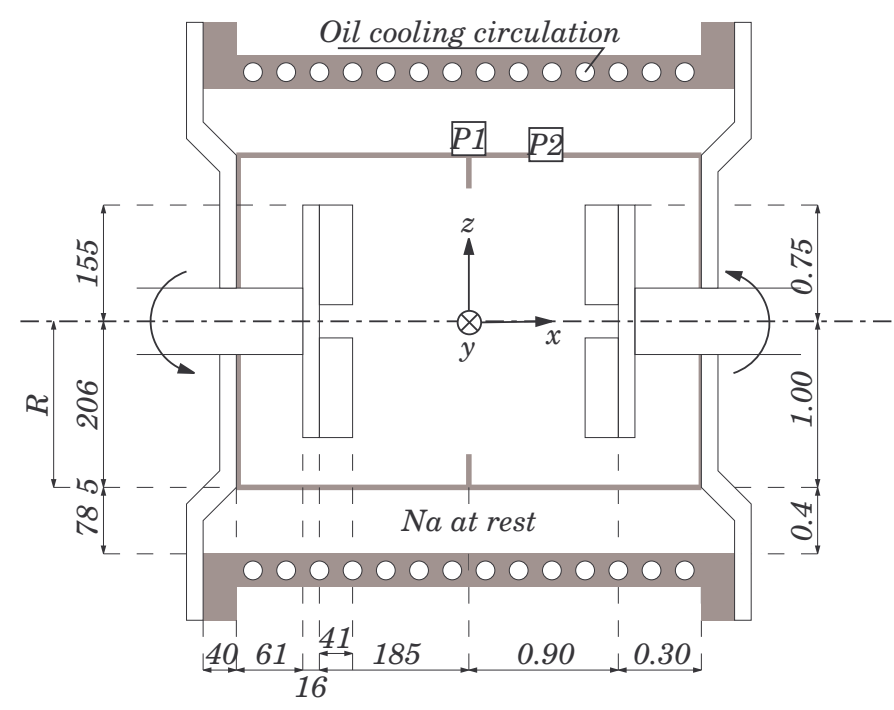

Fig. 1: Sketch of the VKS experiment [5]. The inner and outer cylinders are made of copper (in gray). The dimension are given in millimeter (left) and normalized by the radius of the inner cylinder (right).

quency, the structure of the mean flow (averaged in time) has the following characteristics: the fluid is ejected radially from the disks by the centrifugal force and loops back towards the axis in the mid-plane between the impellers. A strong differential rotation is superimposed on this poloidal flow, which generates a high shear in the mid-plane. We approximate the experimental configuration with impellers of radius $R$ (i.e. extending up to the inner cylinder boundary). We use cylindrical coordinates $(s, \phi, z)$. In this cylindrical domain $([0,1] \times[0,2 \pi] \times[-1,1])$, the flow is well described by the following analytical expression

$$
\boldsymbol{u}=\boldsymbol{\nabla} \times\left(\psi \mathbf{e}_{\phi}\right)+s \omega \mathbf{e}_{\phi},
$$

with angular velocity $\omega$ and recirculation $\psi$ respectively given by

$$
\begin{gathered}
\omega=4 \varepsilon(1-s) \sin \left(\frac{\pi}{2} z\right), \\
\psi=\frac{s}{2}(1-s)^{2}(1+2 s) \sin (\pi z) .
\end{gathered}
$$

This flow has been shown to generate a similar kinematic dynamo as the one computed using the experimentally measured mean flow [9]. In the above expression, $\varepsilon$ is a parameter controlling the ratio between the poloidal and toroidal components of the flow. We take $\varepsilon=0.7259$ as in previous numerical studies using this flow, in order to minimize the critical magnetic Reynolds number for dynamo threshold $[9,10]$.

Although we are aware that the experimentally observed dynamo cannot be captured with a kinematic calculation using the mean flow alone, we use this simple model here in order to study the effect of the magnetic boundary conditions on the dynamo threshold. We hope that the qualitative behaviors will be unchanged with other dynamo modes in the presence of a turbulent flow.
We perform direct numerical simulations of kinematic dynamos, solving the induction equation governing the evolution of the solenoidal magnetic field $\mathbf{B}$

$$
\frac{\partial \boldsymbol{B}}{\partial t}=R m \boldsymbol{\nabla} \times(\boldsymbol{u} \times \boldsymbol{B})+\Delta \boldsymbol{B},
$$

written in dimensionless form, using the diffusive timescale. The magnetic Reynolds number $R m$ is defined as $R m=\mu_{0} \sigma R U_{\max }$, where $R$ is the cylindrical radius of the domain of the flow defined by $(1 \mathrm{a}-\mathrm{c})$ and $U_{\max }$ is the peak velocity of the flow.

Equation (2) with the flow given by (1a-c) is solved using a finite volume code adapted from [11]. To circumvent the severe CFL restriction induced by cylindrical coordinates, we ensure numerical stability near the axis using a low pass Fourier filter in the $\phi$-direction. Also a centered second order scheme has been preferred fo to an up-wind scheme to discretize the inductive term, as resistive effects are here important enough to regularise the solution. As in [11], we ensure that $\boldsymbol{\nabla} \cdot \boldsymbol{B}=0$ is exactly satisfied using a constraint transport algorithm. The finite volume solver is fully three-dimensional. We have not used the decoupling of Fourier modes in the $\phi$-direction. The initial magnetic field obviously needs to satisfy the divergence-free constraint as well as the boundary conditions. In practice, an arbitrary divergent free field is initialized away from all boundaries.

We investigate several types of magnetic boundary conditions. The classical approach is to use insulating boundaries, matching the internal magnetic field to the vacuum magnetic potential. The continuity of the magnetic field results in a non-local set of boundary conditions, which can be expressed via a "Neumann to Dirichlet" operator. We rely here on such an approach, using the boundary element formalism, as introduced in [12].

We investigate the effects introduced by using ferromagnetic boundaries. This boundary condition can be expressed in a local form in the limit of infinite permeability. Jump conditions at a boundary between media of different magnetic permeability are well established

$$
\begin{gathered}
\left.\boldsymbol{B} \cdot \mathbf{n}\right|_{2}=\left.\boldsymbol{B} \cdot \mathbf{n}\right|_{1}, \\
\boldsymbol{B} \times\left.\mathbf{n}\right|_{2}=\frac{\mu_{2}}{\mu_{1}} \boldsymbol{B} \times\left.\mathbf{n}\right|_{1},
\end{gathered}
$$

where subscripts 1 and 2 denote the two different regions and $\mathbf{n}$ is normal to the boundary. Ferromagnetic disks yield $\mu \gg \mu_{0}$, one can therefore reasonably approximate these jump relations, by using boundary conditions on the fluid side of the form

$$
B \times \mathbf{n}=\mathbf{0} .
$$

This set of boundary conditions trivially implies that normal currents vanish. 
We will consider the effect of iron disks in two different ways. The first, and probably simpler, approach is to assume that the field is normal to the disks, namely

$$
\boldsymbol{B} \times \mathbf{e}_{z}=\mathbf{0},
$$

on the top and bottom of the cylinder. This boundary condition is well known in magnetohydrodynamics, in particular in the astrophysical community, and is sufficient to close the system of equations we investigate. Another, and more subtle approach, allows to take into account the effect of the blades on the disks. Assuming that the field becomes normal to radial blades as it approaches the end of the cylinder yields

$$
\boldsymbol{B} \times \mathbf{e}_{\phi}=\mathbf{0} .
$$

This is an extremely simplified approach which does not take into account each individual blade, but accounts for their average effect on the large scale field. This set of boundary conditions is far less common, and deserves some care to ensure it provides the required constraints on the field. The solenoidal nature of magnetic field $(\boldsymbol{\nabla} \cdot \boldsymbol{B}=0)$, together with the fact that $B_{s}$ is identically zero on the boundary, then imply

$$
\frac{\partial B_{\phi}}{\partial \phi}=-s \frac{\partial B_{z}}{\partial z} .
$$

This yields the solvability condition

$$
\oint_{0}^{2 \pi} \frac{\partial B_{z}}{\partial z} d \phi=0 .
$$

As the flow we consider here is axisymmetric, modes in the $\phi$-direction decouple, and we know from Cowling's theorem that the magnetic eigenmode cannot be axisymmetric. The solvability condition is therefore obviously satisfied (this would not be the case for a non-linear simulation).

For non axisymmetric modes, one can write

$$
\oint_{0}^{2 \pi} B_{\phi} d \phi=0
$$

which together with (8) determines the $B_{\phi}$ field completely. The numerical implementation is two fold, we first compute

$$
B_{\phi}^{\prime}(\phi)=-s \oint_{0}^{\phi} \frac{\partial B_{z}}{\partial z} d \phi,
$$

up to an arbitrary constant. Then we correct this function setting by the constant to meet (10).

This implementation is mathematically consistent and maintains the divergence free property of the magnetic field. We shall however stress again that this very idealized condition can only be used here because the modes decouple in the azimuthal direction and the axisymmetric mode cannot be unstable.
The convergence of the numerical implementation has been carefully validated comparing simulations at different resolutions. We report results obtained with a resolution of $200 \times 200 \times 256$ points. When an additional domain of sodium at rest is included, we use the same resolution in the inner flow domain defined by (1a-c), which leads for the full domain to 240 points in the radial direction and 250 points in the $z$-direction to maintain a uniform accuracy.
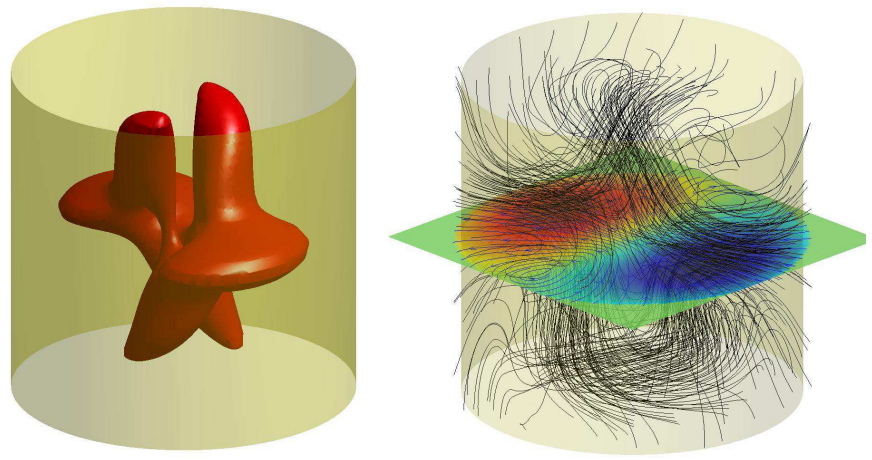

Fig. 2: The magnetic eigenmode obtained with potential boundary conditions. The rotation axis is vertical. An isosurface of the magnetic energy ( $25 \%$ of the maximum value) is represented on the left. Magnetic field lines are plotted on the right.

As in previous studies, all simulations yield magnetic eigenmodes with an $m=1$ azimuthal symmetry. The structure of this eigenmode essentially corresponds to an equatorial dipole. This mode is represented on Figure 2

Previous numerical studies $[13,14]$ compared the threshold values in configurations including and excluding the effect of fluid behind the disks. We reproduce here a similar behaviour of the threshold: using insulating boundary conditions directly on the disks yields a threshold value $R m_{c}=63$. This last value is in good agreement with [14]. Adding a layer of thickness 0.25 of fluid at rest between each disk and insulating boundaries increases the dynamo onset up to $R m_{c}=72$. This is illustrated on Figure 3 , Simulations, not discussed here, show that if the sodium behind the disks is not at rest, the threshold is even further increased.

When we consider the case of ferromagnetic disks, the threshold for dynamo action is $R m_{c}=60.5$ for a magnetic field normal to the disks and $R m_{c}=58$ for a magnetic field in the $\phi$-direction. These two thresholds are very close and lower than in the case of a potential field (see also Figure 3). We thus observe that the high permeability boundary condition on the disks appears to do more than just screening the inhibition of electromagnetic induction due to the flow behind the disks.

Implementation of a cylindrical layer of sodium at rest on the side can easily be included in the simulations. Since all electrical quantities are continuous, and in particular there is no jump in the conductivity, this does not involve 


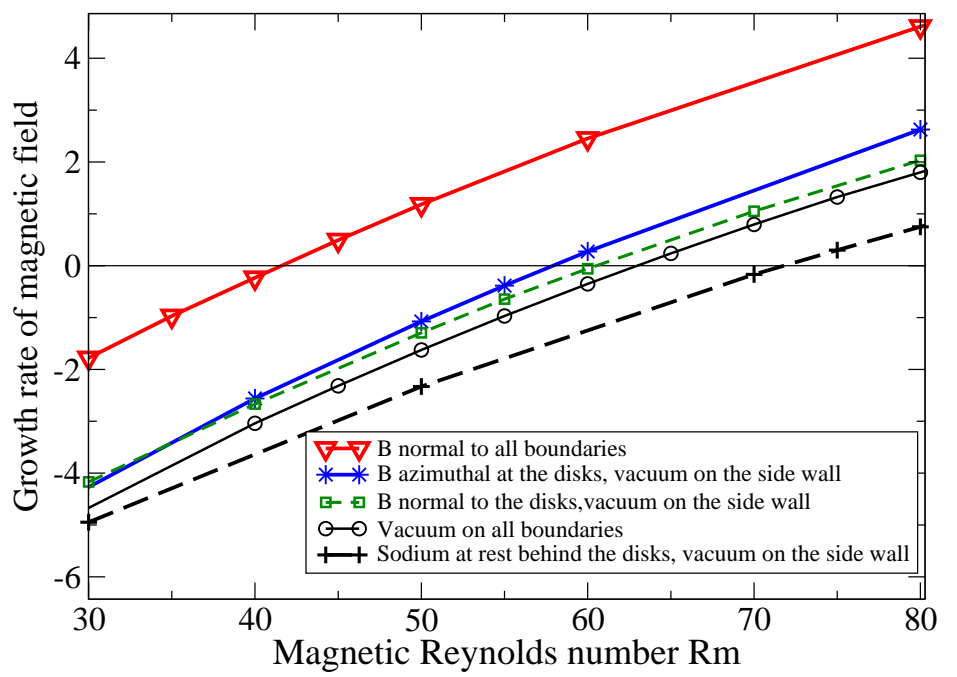

Fig. 3: Growth rate of the magnetic field as a function of $R m$ for different types of boundary conditions. We can see that replacing sodium at rest behind the disks by vacuum leads to a reduction of the dynamo threshold. Moreover, ferromagnetic conditions lead to additional reduction of threshold (about 10\% for azimuthal conditions). Note that using high magnetic permeability boundary conditions on the disks but also on the side yields the best configuration, with an onset at $R m_{c}=41.5$.

any extra boundary condition. In some sense, the model assumes a continuous fluid whose velocity field goes to zero outside a given domain. Table 1 reports critical parameters calculated for different combinations of boundary conditions on the side and on the disks. The sodium at rest leads to an important reduction of dynamo thresholds when it is added on the side. It is known $[10,13,14]$ that this effect increases with the width of the layer, but saturates relatively rapidly. In all the simulations presented, the width of the layer of sodium at rest on the side is set to 0.4 in order to study a configuration close to the VKS experiment. This positive impact on the onset appears to be independent of the boundary conditions on the disk.

Finally, observing the strong effect produced by ferromagnetic disks, it is tempting to investigate the effect of high permeability material for the entire vessel. We thus perform simulations for which the magnetic field is normal to all boundaries (see Figure 3). This configuration appears to be the most efficient and it leads to a critical magnetic Reynolds number $R m_{c}=41.5$. It is clear from Table 1 that in the presence of sodium at rest, ferromagnetic boundaries still yield a strong reduction of the onset, despite the fact that the boundary is now remote from the flow domain. No significant modification of the global magnetic structure can be observed in our simulations when we compare ferromagnetic and vacuum bound-

\begin{tabular}{|l|c|c|c|c|}
\hline disks & $N a \& B_{n}$ & $B_{n}$ & $N a \&-\nabla \Phi$ & $-\nabla \Phi$ \\
\hline$B_{\phi}$ & 39 & 41 & 45 & 58 \\
\hline$B_{n}$ & 40 & 41.5 & 45.5 & 60.5 \\
\hline$-\nabla \Phi$ & 44 & 43.5 & 48.5 & 63 \\
\hline$N a \& B_{\phi}$ & 46 & 47 & 53 & 71 \\
\hline$N a \& B_{n}$ & 47 & 47.5 & 53.8 & 71.5 \\
\hline$N a \&-\nabla \Phi$ & 47.5 & 48 & 54.7 & 72 \\
\hline
\end{tabular}

Table 1: Dynamo thresholds for different types of boundary conditions on the disks and on the sides of the cylinder. $B_{n}$ and $B_{\phi}$ denote ferromagnetic boundaries (respectively normal to the disks and normal to the blades), $-\nabla \Phi$ denotes the vacuum condition and $\mathrm{Na}$ indicates the presence of a layer of sodium at rest between the flow and the boundary. For the disks, cases $N a \& B_{\phi}, N a \& B_{n}$ or $N a \&-\nabla \Phi$ correspond to the presence of a layer of sodium at rest, outside which the relevant boundary condition is applied. The sodium lies behind the disks, and the boundary conditions are implemented on the lids.

ary conditions. The magnetic eigenmodes in the bulk are similar with both sets of boundary conditions.

The effect of the electrical conductivity of the boundaries on the dynamo threshold has been studied since a long time [15]. It has been shown that the addition of a layer of electrically conducting fluid at rest around the flow can either decrease or increase the threshold [16]. This has also been observed in the case of von Kármán flows, depending on the location of the layer at rest [13,14]. Thus, general rules for the dependence of the dynamo threshold on the electrical conductivity of the boundary do not seem to exist. Such may not be the case with the magnetic permeability of the bounding domain. Previous studies on the Ponomarenko dynamo with high magnetic permeability boundaries have displayed a decrease of the threshold $[17,18]$. A similar improvement has been observed in the case of convectively driven dynamos [19]. In the present study, we find that boundaries with a high magnetic permeability always decrease the dynamo threshold whatever their location.

Two important aspects of the VKS experiment are not taken into account in the present study. First, the magnetization of iron can lead to an additional amplifying factor of the dynamo as discussed in [7]. However, the coercitive field of pure iron being much smaller than the fields generated by the dynamo, the iron disks do not impose any permanent magnetization. Reversals of the generated magnetic field are indeed observed [20]. Second, the geometry of the magnetic field generated in the VKS experiment differs from the one computed from the axisymmetric mean flow. Non axisymmetric velocity fluctuations generate a poloidal field with a dominant axial dipole, together with a strong azimuthal component. Thus the poloidal field is roughly normal to the disks, whereas the toroidal field is normal to the blades. It would therefore be of interest to check whether the dynamo threshold can be reached by using only ferromagnetic disks or blades. These two cases 
would correspond respectively to our boundary conditions (6) and (7).

Another interesting set of modifications suggested by this numerical study, would be to replace the copper side wall by a ferromagnetic one in the VKS experiment. If the experimentally realised dynamo mode behaves similarly to the simulations, we expect the threshold to be even lower in such configuration than that associated with only ferromagnetic disks and blades.

$* * *$

\section{REFERENCES}

[1] See for instance, MoffatT H.K., Magnetic field generation in electrically conducting fluids (Cambridge University Press, Cambridge) 1978; Dormy E. and SowArd A.M., Mathematical Aspects of Natural Dynamos (CRC-press) 2007.

[2] Stieglitz R. and Müller U., Phys. Fluids, 13 (2001) 561.

[3] Gailitis A. et al., Phys. Rev. Lett., 86 (2001) 3024.

[4] Roberts G. O., Phil. Trans. Roy. Soc. London A, 271 (1972) 411; Ponomarenko Yu. B., J. Appl. Mech. Tech. Phys., 14 (1973) 775.

[5] Monchaux R. et al., Phys. Rev. Lett., 98 (2007) 044502.

[6] Marié L. et al., Eur. Phys. J. B, 33 (2003) 469.

[7] Pétrélis F., Mordant N. and Fauve S., Geophys.Astrophys. Fluid Dyn., 101 (2007) 289.

[8] Bourgoin M. et al., Phys. Fluids, 16 (2004) 2529.

[9] Marié L., Normand C. and Daviaud F., Phys. Fluids, 18 (2006) 017102.

[10] Ravelet F. et al., Phys. Fluids, 17 (2005) 117104.

[11] Teyssier R., Fromang S. and Dormy E., J. Comp. Phys., 218 (2006) 44.

[12] Iskakov A., Descombes S., and Dormy E., J. Comp. Phys., 197 (2004) 540; Iskakov A. and Dormy E., Geophys. Astrophys. Fluid Dyn., 99 (2005) 481.

[13] Stefani F. et al., Eur. J. Mech. B, 25 (2006) 894.

[14] Laguerre R. et al., CRAS Mécanique, 334 (2006) p.593598.

[15] Bullard E. C. and Gubbins D., Geophys. Astrophys. Fluid Dyn., 8 (1977) 43.

[16] Kaiser R. and Tilgner A., Phys. Rev. E, 60 (1999) 2949.

[17] Marty P., Ajakh A. and Thess A., Magnetohydrodynamics, 30 (1995) 474.

[18] Avalos-Zuniga R., Plunian F. and Gailitis A., Phys. Rev. E, 68 (2003) 066307.

[19] Morin V., PhD Thesis, University Paris VII, (2005) Appendix C.

[20] Berhanu M. et al., Europhys. Lett., 98 (2007) 59001. 